\title{
Complete Pathologic Response After Concurrent Treatment with Pembrolizumab and Radiotherapy in Metastatic Colorectal Cancer: A Case Report
}

This article was published in the following Dove Press journal: OncoTargets and Therapy

\author{
Jian Yang' \\ Feng $\mathrm{Bi}^{2}$ \\ Hongfeng Gou' \\ 'West China Hospital, Sichuan University, \\ Chengdu, Sichuan Province, 6I004I, \\ People's Republic of China; ${ }^{2}$ Department \\ of Medical Oncology, Laboratory of \\ Molecular Targeted Therapy in Oncology, \\ State Key Laboratory of Biotherapy, West \\ China Hospital, Sichuan University, \\ Chengdu, Sichuan Province, 61004I, \\ People's Republic of China
}

Correspondence: Feng $\mathrm{Bi}$

Department of Medical Oncology, Laboratory of Molecular Targeted

Therapy in Oncology, State Key Laboratory of Biotherapy, West China Hospital, Sichuan University, 37\# Wai Nan Guoxue Road, Chengdu, Sichuan Province, 6I004I, People's Republic of China

Tel +86-28-85423609(O)

Fax +86-28- 85423609

Email bifeng@scu.edu.cn

Hongfeng Gou

West China Hospital, Sichuan University,

37\# Wai Nan Guoxue Road, Chengdu,

Sichuan Province, 61004I, People's

Republic of China

Email joan_goul977@I63.com

\begin{abstract}
Due to specific genetic characteristics, therapeutic options for colorectal cancer (CRC) with DNA mismatch repair deficient (dMMR)/microsatellite instability-high (MSI-H) are limited. Although programmed death 1 (PD-1) blockade has been shown to be highly effective therapy for dMMR/MSI-H CRC, there is a need to develop new therapeutic paradigms to further improve survival rates of patients with dMMR/MSI-H CRC. So far, there is no case report on the use of immunotherapy combined with radiotherapy (RT) for the treatment of dMMR/MSI-H metastatic CRC (mCRC). Here, we report a 64-year-old patient diagnosed with mCRC who experienced a complete pathological response (pCR) after successfully conversion treatment with pembrolizumab and RT, and remains to be tumorfree during a follow-up of 11 months while off therapy. Immunohistochemical staining for MLH1, MSH2, MSH6, and PMS2 on the intestinal biopsy samples revealed loss of MLH1 and PMS2 protein expression. The present case report adds to the limited data on the safety and effectiveness of local RT combined with immunotherapy for patients with dMMR/MSI$\mathrm{H}$ mCRC. This combination therapy appears to be a potential treatment for dMMR/MSI-H mCRC and deserves further exploration.
\end{abstract}

Keywords: immunotherapy, radiotherapy, pembrolizumab, pathological complete response, colorectal cancer

\section{Introduction}

Colorectal cancer (CRC) is one of the most common and lethal cancers in the world. ${ }^{1}$ Approximately a quarter of CRC cases are diagnosed at an advanced stage. ${ }^{2}$ 5-fluorouracil (5-FU)-based chemotherapy combinations are treatment standard for metastatic CRC (mCRC). ${ }^{3}$ However, evidence suggests that patients with DNA mismatch repair deficient (dMMR)/microsatellite instability-high (MSI-H) mCRC are less responsive to conventional chemotherapy and have poorer prognosis relative to those with mismatch repair-proficient (pMMR) or microsatellite-stable (MSS) disease. ${ }^{4}$ Programmed death 1 (PD-1) blockade has emerged as a highly effective therapy for $\mathrm{dMMR} / \mathrm{MSI}-\mathrm{H}$ mCRC. ${ }^{3}$ Past studies show that the PD-1 inhibitors, pembrolizumab and nivolumab, could lead to durable response in patients with previously treated dMMR/MSI-H mCRC. ${ }^{5,6}$ Data from the KEYNOTE-177 study ${ }^{3}$ found that relative standard chemotherapy in patients with $\mathrm{dMMR} / \mathrm{MSI}-\mathrm{H} \mathrm{mCRC}$, pembrolizumab significantly improves progression-free survival (PFS), objective response rate (ORR), and complete response (CR) rate, doubling median PFS from 8.2 months to 16.5 months, extending ORR from $33.1 \%$ 
to $43.8 \%$, and increasing CR rate from $3.9 \%$ to $11.1 \%$. Additionally, relative to chemotherapy, PD-1 monoclonal antibody has fewer overall treatment-related adverse events. Based on Phase 3 KEYNOTE-177 trial findings, the Food and Drug Administration (FDA) approved pembrolizumab as first-line treatment in patients with unresectable or metastatic dMMR/MSI-H CRC. ${ }^{7}$ Despite remarkable advances in immune checkpoint inhibitor (ICI) therapy, more than half of the patients do not respond to immune monotherapy. ${ }^{8}$ To maximize response rates to ICIs, combined immunotherapies, both systemic and local, are being developed. The CheckMate142 study increased ORR to $69 \%$ and $\mathrm{CR}$ rate to $13 \%$ by combining ipilimumab, a cytotoxic T-lymphocyte antigen-4 (CTLA-4) inhibitor, with nivolumab, a PD-1 inhibitor. ${ }^{6}$ Thus, combining nivolumab with low-dose ipilimumab may be a new firstline treatment option for patients with dMMR/MSI-H mCRC. However, overlapping toxicities from the dual immunotherapy remains a challenge. Moreover, treatment modalities for immunotherapies like atezolizumab (Trial identifier: NCT02912559; NCT02997228; NCT01633970) in combination with chemotherapy are also being explored (Clinical trial details can be accessed at ClinicalTrials.gov database). A growing body of preclinical and clinical data suggests that the immune system is a critical determinant of response to radiotherapy (RT). ${ }^{9,10} \mathrm{RT}$ is used to control local disease but recent technological advances in RT delivery markedly enhanced local tumor control and in some cases reduced metastatic burden. ${ }^{11}$ Some reports and studies indicate that in various cancers, including metastatic melanoma, ${ }^{12}$ non-small cell lung cancer, ${ }^{13}$ and prostate cancer, ${ }^{14}$ local RT in combination with immunotherapy may improve therapeutic responses. While there might be synergistic or complementary effect between the two modalities, little is known of the underlying mechanisms. ${ }^{15}$ However, to the best of our knowledge, there are no case reports on immunotherapy-RT combination in patients with dMMR/MSI-H mCRC. Here, we describe the course of treatment in an interesting and challenging case of a patient with dMMR/MSI-H mCRC who underwent successful surgical resection and achieved complete pathological response (pCR) after immunotherapy-RT combined treatment, suggesting that patients with dMMR/MSI-H mCRC may benefit from combined immunotherapy-RT treatment modalities.

\section{Case Presentation}

A 64-year-old male with a history of abdominal pain and bloody stool for several days was admitted into the clinic in June 2019. He then underwent colonoscopy biopsy, which revealed adenocarcinoma (Figure 1A). Abdominal contrastenhanced computed tomography $(\mathrm{CT})$ revealed that ascending colon cancer had broken through the serosa with multiple mesenteric and retroperitoneal lymph node metastases, inferior vena cava and right ureter involvement, and right kidney and right renal arteriovenous invasion (July 4, 2019) (Figure 2).

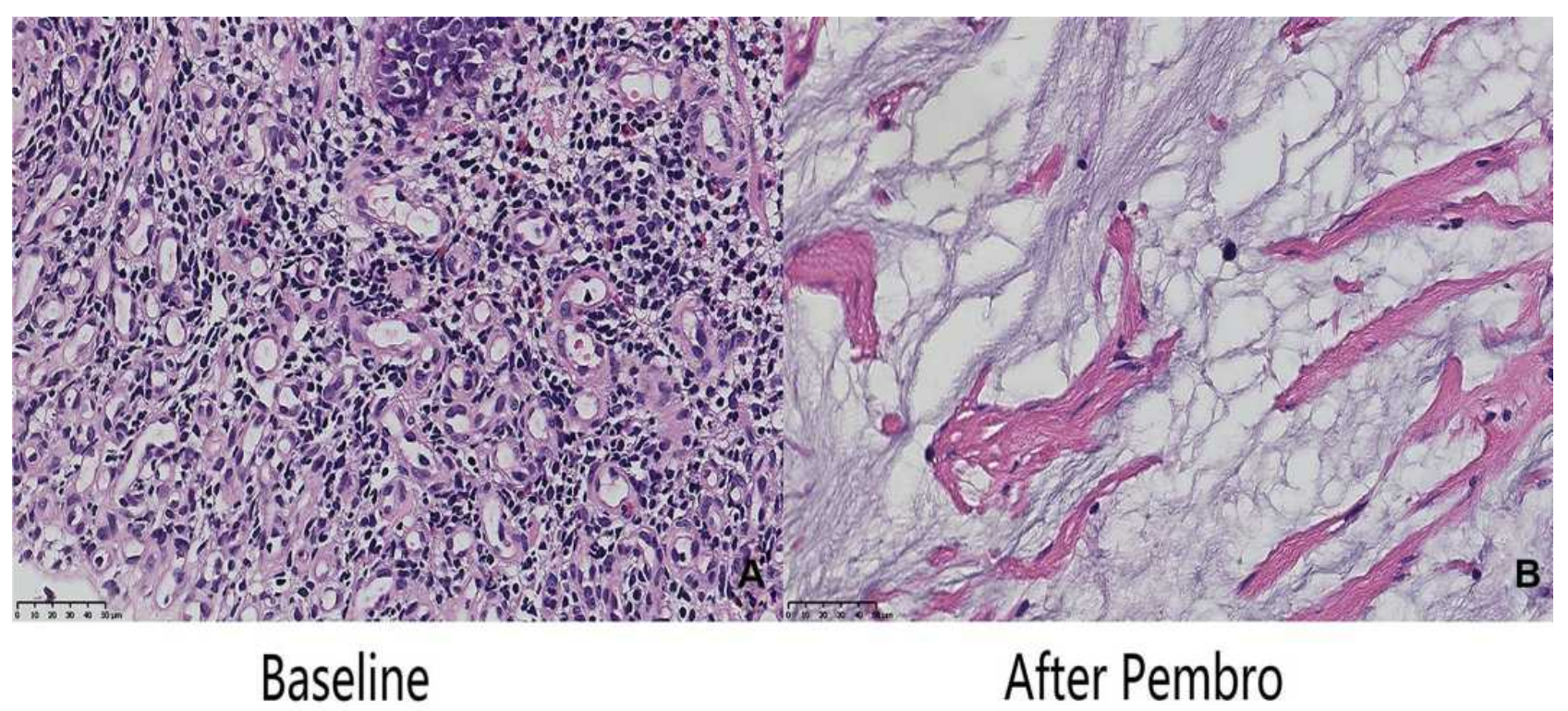

Figure I (A) The pathological findings of colonoscopy biopsy. It shows that atypical cells are arranged in an irregular glandular tube-like arrangement, with large and deeply stained nuclei and obvious mitosis (H\&E, 40x). (B) The microscopic findings of the resected specimen. There is only mucus but no exact cancer cells were found (H\&E, 40x). 


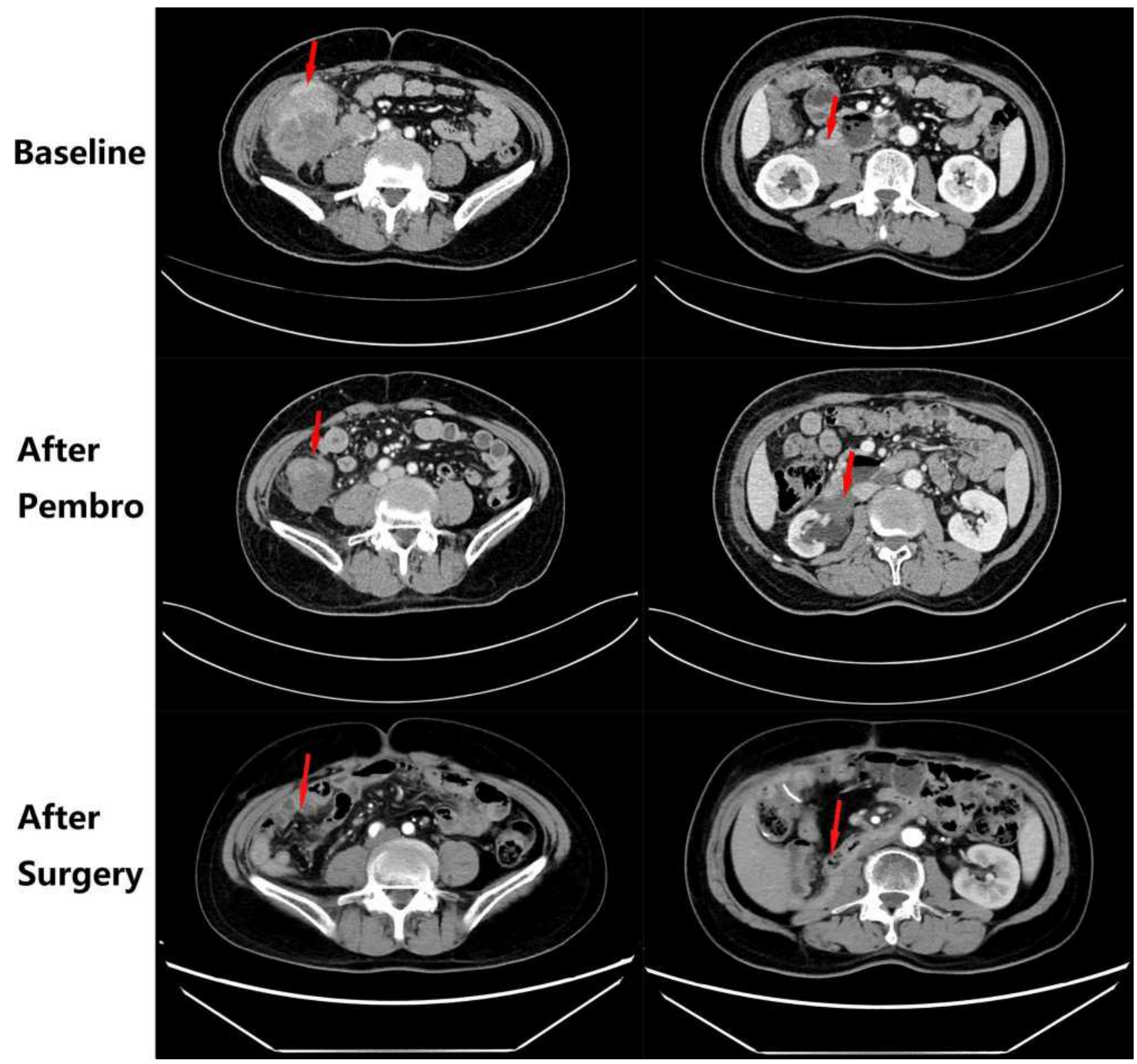

Figure 2 The intestinal and kidney mass treatment effect images of patient at baseline, after pembrolizumab and after surgery, respectively. The red arrow indicates the lesion.

Based on clinical tumor-node-metastasis (cTNM), the cancer was classified as cT4N2M1. Mutation analysis revealed wildtype KRAS, NRAS, and BRAF. Immunohistochemical staining for MLH1, MSH2, MSH6, and PMS2 on the patient's archival intestinal biopsy samples revealed loss of MLH1 and PMS2 expression (Figure 3). The case was evaluated by a multidisciplinary team (MDT) that concluded that the lesions were potentially resectable and started treatment with conversion chemotherapy. Three cycles (2019-8-15, 2019-9-2, 20199-26) of leucovorin (400 $\mathrm{mg} / \mathrm{m} 2)$, 5-fluoropyrimidine (2400 $\mathrm{mg} / \mathrm{m} 2)$, irinotecan $(180 \mathrm{mg} / \mathrm{m} 2)$ and oxaliplatin $(85 \mathrm{mg} / \mathrm{m} 2)$ (FOLFOXIRI) resulted in stable disease (SD).
Tumor response was assessed using response evaluation criteria in solid tumors (RECIST) guidelines (version1.1). In August, the patient's serum carcinoembryonic antigen (CEA) levels also rose from $10.18 \mathrm{ng} / \mathrm{mL}$ to $21.7 \mathrm{ng} / \mathrm{mL}$ (normal range: $0-3.4 \mathrm{ng} / \mathrm{mL}$ ). Based on the facts that immunohistochemistry showed the patient had locally confirmed MSI-H status, that he had received 1 prior line of therapy (FOLFOXIRI), and that the unsatisfactory efficacy of previous first-line regimen, the MDT developed a treatment strategy combining immunotherapy (pembrolizumab) and intensity-modulated RT to explore the potential benefit of a second intervention. After four cycles of pembrolizumab (200mg every 3 weeks) and concurrent 25 


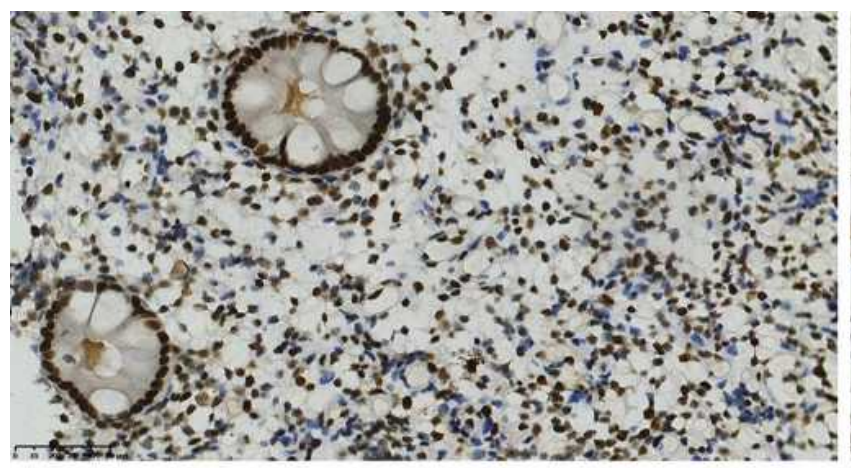

MLH1 (40x)

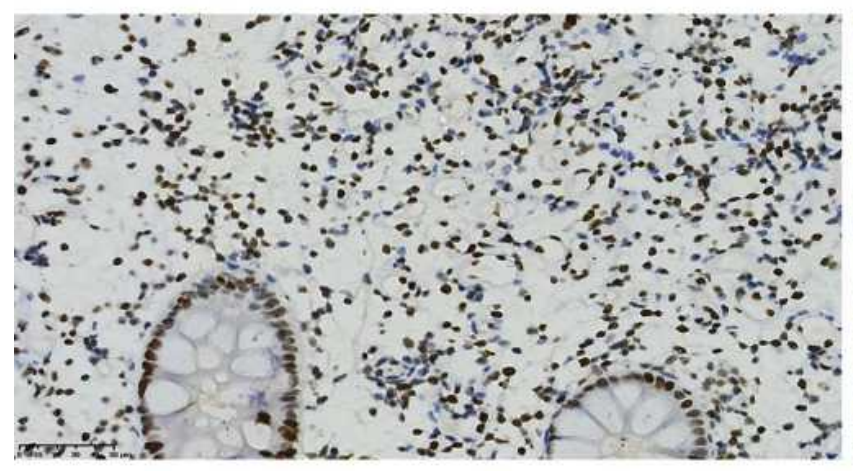

MSH6 (40x)

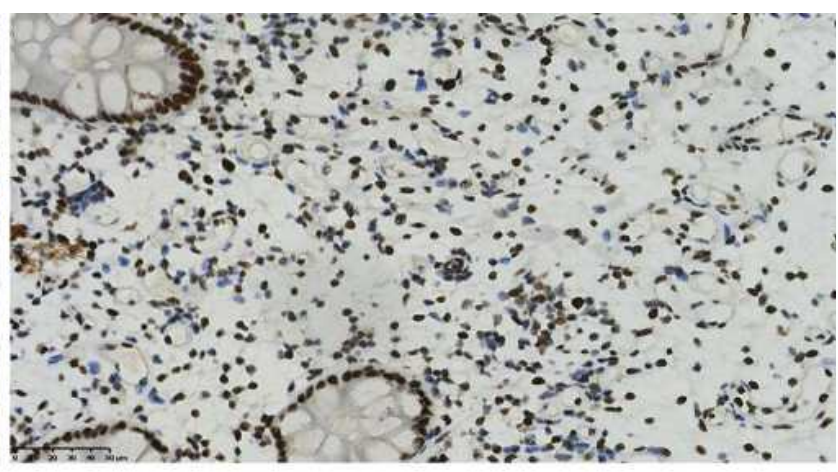

MSH2 (40x)

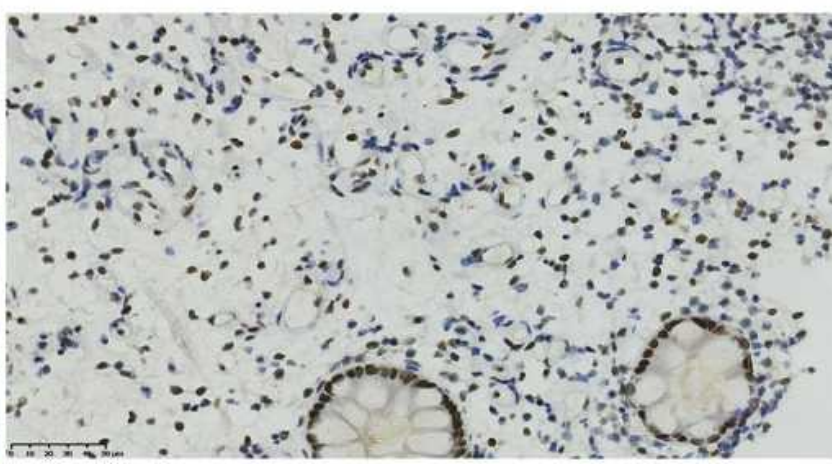

PMS2 (40x)

Figure 3 Immunohistochemical staining for MLHI, MSH2, MSH6, and PMS2 on the intestinal biopsy samples reveal loss of MLHI and PMS2 protein expression (40x).

radiation treatments (metastatic lesions in retroperitoneal lymph nodes were also included in the RT field that included colon lesions, right kidney, and retroperitoneal lymph nodes), with a total dose of 45Gy (from 2019-10 to 2020-1, Figure 4) administered in conversion setting, CT scanning revealed that the patient had robust partial remission (PR) (Figure 2). CEA also declined to the normal range (Figure 5). Upon comprehensive assessment, the MDT concluded that the patient could undergo synchronous resection during surgery. Intraoperative dissection showed that the neoplasm was located in the ileocecal valve, involved the intestine for a cycle, and invaded the back of the right kidney parenchyma. Thus, the patient was treated with expansive radical right hemicolectomy + right nephrectomy + retroperitoneal lymphadenectomy on March 19, 2020 (Figure 6). Postoperative pathological examination revealed complete primary and secondary tumor response without evidence of malignancy. Microscopic examination found only mucus but no viable cancer cells in the whole intestinal layer, perianal fat, fibrous tissue around the ureter, and lymph nodes around the vena cava (Figure 1B). The renal parenchyma, ureteral stump, and the distal and proximal incisions of the colon were all cancer-free. Tumor response to radiochemotherapy was complete response of Tumor Regression Grade 0 (TRG 0). No immune-related adverse events were recorded in the treatment period. The patient recovered well after surgery and received no anticancer regimens. He continues outpatient follow up 11 months postoperatively and no colon cancer recurrence has been observed through CT evaluation and CEA detection. The patient had a history of hypertension for more than 10 years and regularly took antihypertensive drugs. He had no known history of colorectal carcinoma or related gastrointestinal diseases. Appendectomy and cholecystectomy were performed for "acute appendicitis" and "cholecystolithiasis".

\section{Discussion}

ICI directed against PD-1 proteins is highly effective and now a first-line standard-of-care therapy for patients with dMMR/MSI-H mCRC. ${ }^{16}$ Based on impressive performance against advanced disease, clinical trials are evaluating the role of ICI in dMMR/MSI-H CRC in the adjuvant and neoadjuvant setting. The NICHE study ${ }^{16}$ found pathological response in all $20 \mathrm{dMMR} / \mathrm{MSI}-\mathrm{H}$ patients who received ipilimumab + nivolumab, with 12 pCRs. ${ }^{16}$ 


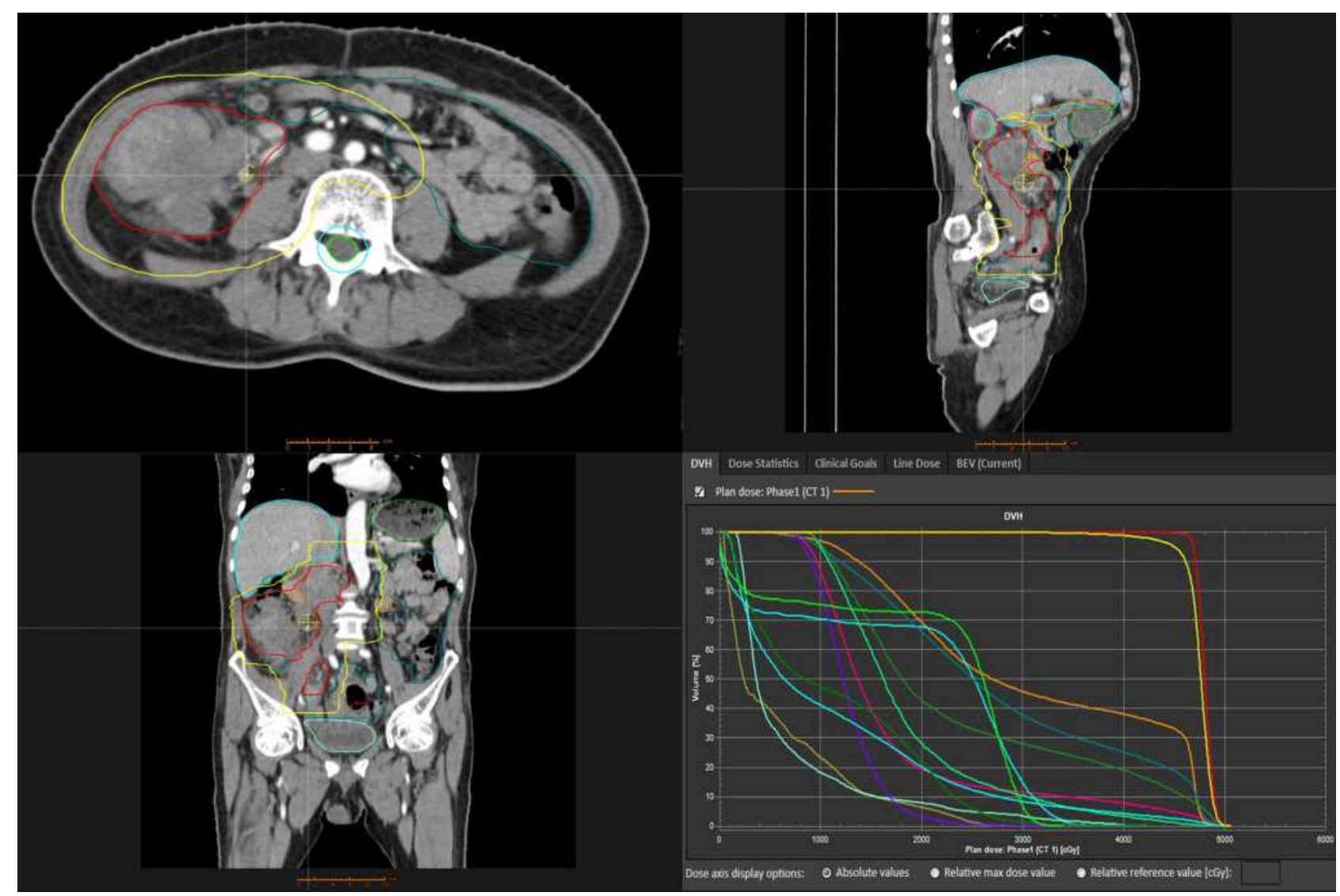

Figure 4 The irradiated gross tumor volume and the radiation dose plan.

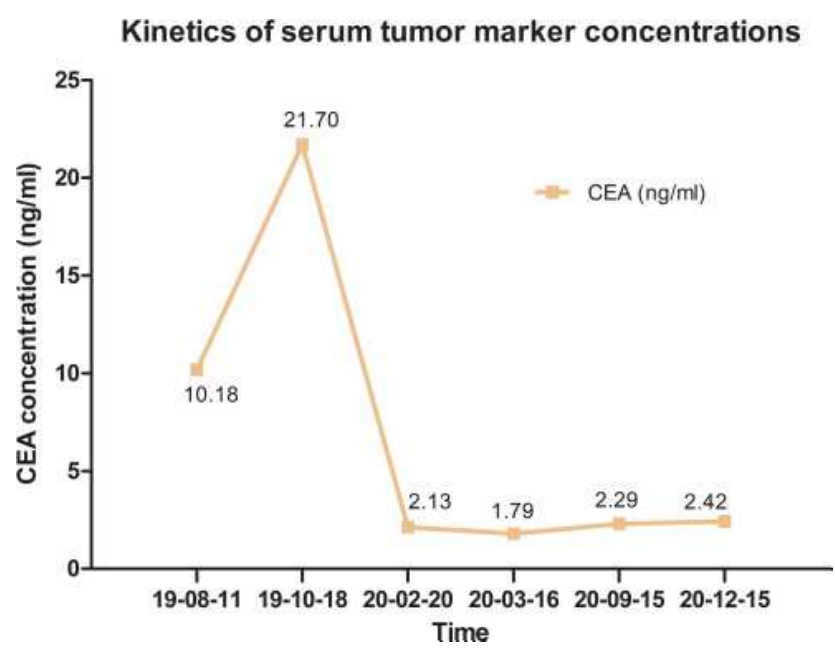

Figure 5 Dynamic change curves of CEA in peripheral blood during the whole treatment process.

Abbreviation: CEA, carcinoembryonic antigen.

Despite advances in ICI therapies in CRC treatment, $>50 \%$ of patients with dMMR/MSI-H mCRC do not respond. ${ }^{8}$ Thus, strategies for expanding PD-1 immunotherapy benefits are underway to improve dMMR/MSI-H mCRC

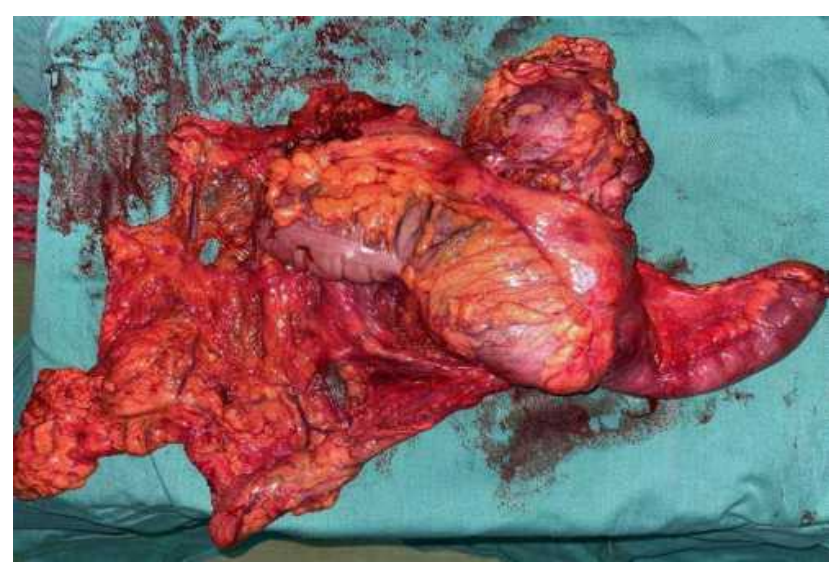

Figure 6 Resection samples of intestines and kidneys.

outcomes. The PD-1/PD-L1 axis is a potent inhibitor of immune activation, particularly through inhibition of effector T-cell function. ${ }^{17}$ Therapeutic blockade of the axis can normalize T-cell effector function and reset immunity. ${ }^{18}$ Hence, the way to improve PD-1 immunotherapy may involve combinations with treatments that induce effector T-cell inflammation. ${ }^{19}$ RT has promising 
potential for use in combination therapy. Because RT has local and systemic effects on the immune system, its combination with immunotherapy may maximize antitumor immune response, inducing durable disease control. ${ }^{20}$ Radiation may improve immunotherapy through increased tumor antigen release, activation of innate immune pathways, increased T-cell infiltration, augmented antigen presentation, and modulation of immunosuppressive cells. ${ }^{8}$ Moreover, preclinical observations show that ionizing radiation can upregulate PD-L1 expression and reduce tumor burden, which may improve ICI efficacy. ${ }^{8}$ Some clinical data also confirm that RT may sustainably maintain host immunity in favorable immune environments with ICI treatment as well as temporary immune response. ${ }^{21}$ Abscopal effect is worth mentioning although not apply to this case. It refers to tumor responses at sites distant from the irradiated volume and it is a rare event. ${ }^{22}$ The specific mechanism underlying abscopal responses is unclear and relatively unexplored in clinical settings. It is thought that it may result from the induction of a systemic immune response triggered by combined immunostimulatory effects of RT and immunotherapy. ${ }^{22}$

Here, we present a case in which the patient profoundly responded to immuno-radiotherapy with good tolerance to the treatment regimen. The final outcome is highly encouraging and the pCR of primary and secondary lesions makes this case unique. To our knowledge, this is the first report of RT combination with immunotherapy to achieve pCR in second-line treatment of dMMR/MSI-H mCRC. In view of findings from the KEYNOTE-177 trial, $11.1 \%$ of dMMR/MSI-H mCRC patients achieved complete response using pembrolizumab alone. We could not precisely assess the contribution of RT in this case, but it is clear that the combined therapy was safe and effective in this patient. Nonetheless, there are still many points that are yet to be clarified. First, although the patient postoperative pathology confirmed that response to the combination treatment was pCR, preoperative $\mathrm{CT}$ assessment of the lesions is still obvious. Raising the question if current imaging techniques like $\mathrm{CT}$, and RECIST evaluation criteria are suitable for evaluating immunotherapy efficacy and whether other imaging techniques like Magnetic Resonance Imaging (MRI) or Positron Emission Tomography-Computer Tomography (PET-CT) are better choices. Secondly, optimal RT delivery for immune activation is complex and many challenges remain for this combination modality, including determination of optimal RT dosing/ fractionation, administration of optimal timing or sequencing for these two treatment protocols, safety of combination therapy, and identification of biomarkers for predicting treatment efficacy. ${ }^{23,24}$ Finally, specific mechanism underlying immunotherapy-RT cooperation is unknown and numerous clinical trials (Trial identifier: NCT 04558684; NCT03507699; NCT04663763; NCT04017455) are trying to address this question. Especially, a single arm Phase II clinical trial at MSKCC (NCT02437071) is assessing the response rate of mCRC to combination pembrolizumab and either RT or radiofrequency ablation. We also expect the result of this clinical trial will further shed the light about the additional benefit provided by combining radiotherapy and pembrolizumab instead of monotherapy with pembrolizumab. Findings from these clinical trials will clarify the mechanisms of combination therapy and enable optimization of RT regimens to maximize synergy with immunotherapy.

In conclusion, the combination therapy of PD-1 blockade and RT is a potential treatment strategy for mCRC. Randomized studies are warranted to determine whether RT-PD-1 blockade combination therapy potentiates efficacy.

\section{Ethical Statement}

This study was approved by Ethics Committee of Sichuan University West China Hospital and the patient provided written informed consent prior to the preparation of this case report.

\section{Funding}

This study was supported by grants from 1.3.5 Project for Disciplines of Excellence, West China Hospital, Sichuan University (No. ZYGD18005), the National Natural Science Foundation (No. 81621003), the National Natural Science Foundation of China (No. 81872020), the National Natural Science Foundation of China (No. 82073059), and Science and Technology Department of Sichuan Province (No. 2019YJ0056).

\section{Disclosure}

The authors declare that they have no competing interests.

\section{References}

1. Coupez D, Hulo P, Touchefeu Y, et al. Pembrolizumab for the treatment of colorectal cancer. Expert Opin Biol Ther. 2020;20(3):219-226. doi:10.1080/14712598.2020.1718095 
2. Siegel RL, Miller KD, Goding Sauer A, et al. Colorectal cancer statistics, 2020. CA Cancer J Clin. 2020;70(3):145-164. doi: $10.3322 /$ caac. 21601

3. André T, Shiu KK, Kim TW, et al. Pembrolizumab in microsatellite-instability-high advanced colorectal cancer. $N$ Engl J Med. 2020;383(23):2207-2218. doi:10.1056/NEJMoa2017699

4. Le DT, Durham JN, Smith KN, et al. Mismatch repair deficiency predicts response of solid tumors to PD-1 blockade. Science. 2017;357(6349):409-413. doi:10.1126/science.aan6733

5. Le DT, Kim TW, Van Cutsem E, et al. Phase II Open-Label Study of pembrolizumab in treatment-refractory, microsatellite instability-high/mismatch repair-deficient metastatic colorectal Cancer: KEYNOTE-164. $J$ Clin Oncol. 2020;38(1):11-19. doi: $10.1200 / \mathrm{JCO} .19 .02107$

6. Overman MJ, McDermott R, Leach JL, et al. Nivolumab in patients with metastatic DNA mismatch repair-deficient or microsatellite instability-high colorectal cancer (CheckMate 142): an open-label, multicentre, Phase 2 study. Lancet Oncol. 2017;18(9):1182-1191. doi:10.1016/S1470-2045(17)30422-9

7. FDA approves Merck's Keytruda (pembrolizumab) first-line treatment of patients with unresectable or metastatic MSI-H or dMMR colorectal cancer. News release. Merck. June 29, 2020. Available from: https://www.fda.gov/news-events/press-announcements/fdaapproves-first-line-immunotherapy-patients-msi-hdmmr-metastaticcolorectal-cancer. Accessed March 29, 2021.

8. Khoja L, Butler MO, Kang SP, et al. Pembrolizumab. J Immunother Cancer. 2015;3(1):36. doi:10.1186/s40425-015-0078-9

9. Deng L, Liang H, Burnette B, et al. Irradiation and anti-PD-L1 treatment synergistically promote antitumor immunity in mice. $J$ Clin Invest. 2014;124(2):687-695. doi:10.1172/JCI67313

10. Newton JM, Hanoteau A, Liu HC, et al. Immune microenvironment modulation unmasks therapeutic benefit of radiotherapy and checkpoint inhibition. J Immunother Cancer. 2019;7(1):216. doi:10.1186/ s40425-019-0698-6

11. Mujoo K, Hunt CR, Pandita RK, et al. Harnessing and optimizing the interplay between immunotherapy and radiotherapy to improve survival outcomes. Mol Cancer Res. 2018;16(8):1209-1214. doi:10.1158/1541-7786.MCR-17-0743

12. Postow MA, Callahan MK, Barker CA, et al. Immunologic correlates of the abscopal effect in a patient with melanoma. $N$ Engl J Med. 2012;366(10):925-931. doi:10.1056/NEJMoa1112824

13. Golden EB, Demaria S, Schiff PB, et al. An abscopal response to radiation and ipilimumab in a patient with metastatic non-small cell lung cancer. Cancer Immunol Res. 2013;1(6):365-372. doi:10.1158/ 2326-6066.CIR-13-0115
14. Kwon ED, Drake CG, Scher HI, et al. Ipilimumab versus placebo after radiotherapy in patients with metastatic castration-resistant prostate cancer that had progressed after docetaxel chemotherapy (CA184-043): a multicentre, randomised, double-blind, phase 3 trial. Lancet Oncol. 2014;15(7):700-712. doi:10.1016/S14702045(14)70189-5

15. Sharabi AB, Nirschl CJ, Kochel CM, et al. Stereotactic radiation therapy augments antigen-specific PD-1-mediated antitumor immune responses via cross-presentation of tumor antigen. Cancer Immunol Res. 2015;3(4):345-355. doi:10.1158/2326-6066.CIR-14-0196

16. Chalabi M, Fanchi LF, Dijkstra KK, et al. Neoadjuvant immunotherapy leads to pathological responses in MMR-proficient and MMR-deficient early-stage colon cancers. Nat Med. 2020;26 (4):566-576. doi:10.1038/s41591-020-0805-8

17. Pardoll DM. The blockade of immune checkpoints in cancer immunotherapy. Nat Rev Cancer. 2012;12(4):252-264. doi:10.1038/ $\operatorname{nrc} 3239$

18. Iwai Y, Terawaki S, Honjo T. PD-1 blockade inhibits hematogenous spread of poorly immunogenic tumor cells by enhanced recruitment of effector T cells. Int Immunol. 2005;17(2):133-144. doi:10.1093/ intimm/dxh194

19. Luke JJ, Lemons JM, Karrison TG, et al. Safety and clinical activity of pembrolizumab and multisite stereotactic body radiotherapy in patients with advanced solid tumors. J Clin Oncol. 2018;36 (16):1611-1618. doi:10.1200/JCO.2017.76.2229

20. Grassberger C, Ellsworth SG, Wilks MQ, et al. Assessing the interactions between radiotherapy and antitumour immunity. Nat Rev Clin Oncol. 2019;16(12):729-745. doi:10.1038/s41571-019-0238-9

21. Sato H, Okonogi N, Nakano T. Rationale of combination of anti-PD1/PD-L1 antibody therapy and radiotherapy for cancer treatment. Int $J$ Clin Oncol. 2020;25(5):801-809. doi:10.1007/s10147-02001666-1

22. Mondini M, Levy A, Meziani L, et al. Radiotherapy-immunotherapy combinations - perspectives and challenges. Mol Oncol. 2020;14 (7):1529-1537. doi:10.1002/1878-0261.12658

23. Liu Y, Dong Y, Kong L, et al. Abscopal effect of radiotherapy combined with immune checkpoint inhibitors. J Hematol Oncol. 2018;11(1):104. doi:10.1186/s13045-018-0647-8

24. Turgeon G-A, Weickhardt A, Azad AA, et al. Radiotherapy and immunotherapy: a synergistic effect in cancer care. Med J Aust. 2019;210(1):47-53. doi:10.5694/mja2.12046
OncoTargets and Therapy

\section{Publish your work in this journal}

OncoTargets and Therapy is an international, peer-reviewed, open access journal focusing on the pathological basis of all cancers, potential targets for therapy and treatment protocols employed to improve the management of cancer patients. The journal also focuses on the impact of management programs and new therapeutic

Submit your manuscript here: https://www.dovepress.com/oncotargets-and-therapy-journa agents and protocols on patient perspectives such as quality of life, adherence and satisfaction. The manuscript management system is completely online and includes a very quick and fair peer-review system, which is all easy to use. Visit http://www.dovepress.com/ testimonials.php to read real quotes from published authors. 